\title{
Design of a Multi-Megawatt X-Band Solid State Microwave Switch*
}

\author{
Sami G. Tantawi ${ }^{\dagger}$, Terry G. Lee, Ronald D. Ruth, A. E. Vlieks and Max Zolotorev \\ Stanford Linear Accelerator Center Stanford, CA 94309
}

\begin{abstract}
:
We present design methodology for high power microwave switches. Among all possible applications for such a switch we emphasize the design parameters for application to the pulse compression system associated with the Next Linear Collider. (NLC)[1]. The switch is based on the excitation of a plasma layer within a silicon wafer by either a laser or an electron beam. We investigate problems associated with high power operation of such a switch. Mainly, we explore solutions to the problems of thermal runaway, avalanche breakdown, photo-emission, and secondary emission. Different design methodologies are presented.
\end{abstract}

\section{INTRODUCTION}

Optical control of microwave components have been under investigation for over two decades. A review of the basic ideas and device physics may be found in [2]. Most of the work in that field was done at milli-meter wavelengths around $94 \mathrm{GHz}$ for application with phased array antennas. For this type of applications the devices need not handle large amounts of power. Also, there is not big constrains on the amount of losses that these devices may exhibit. However, the main emphasis in designing these devices was speed. Pico-second switches and phase shifters is reported on the literature [2]. On the other hand, development of optical control of high power DC. switches required a low loss devices and an appropriate design to deal with thermal runaway problems [2]. Activation with electron beam for these DC switches was also considered[3].

To date, there is no active devices that can control and manipulate multi-megawatt microwave signals. Applications that can make use of such a device include, but not limited to, pulse compression systems required to drive linear accelerators (for scientific research such as NLC, for medical applications and for remote sensing) and, broadening the band width of high power sources such as klystrons and magnetrons by actively detuning there cavities (for radar applications). Bulk effects in semiconductors have the potential of producing a working device in that regime. Of course, the design parameters will depend on the application. In the following presentation we will emphasize on the design parameters required for the NLC. Although the ideas presented may be extended to any of these other applications.

We start by the analysis of a symmetric 3-port device. We explore the ability of controlling the coupling between two of the ports by actively changing the termination of the third port. We derive general expression for, power, losses, and peak electric field in that third arm, which we shall call the active arm. In section III. we explore silicon as an active material for

*This work is supported by the U.S. Department of Energy under contract DE-AC03-76SF00515

$\dagger$ Also with Electrical Communications and Electronics Dept. Cairo University, Giza, Egypt. our device. In section IV we explore different operating modes of the device.

\section{MICROWAVE CONTROL WITH A THREE PORT DEVICE.}

Consider the three port device shown in Figure 1. The device is composed from a basic lossless three port device with two similar ports namely, port 1 and port 2 followed by a two port junction cascaded after port 2. Since the scattering matrix $\underline{S}$ of the symmetric 3-port junction is unitary, at certain reference planes we have

$$
\underline{S}=\left(\begin{array}{ccc}
\sin ^{2}\left(\frac{\theta}{2}\right) & -\cos ^{2}\left(\frac{\theta}{2}\right) & \frac{\sin \theta}{\sqrt{2}} \\
-\cos ^{2}\left(\frac{\theta}{2}\right) & \sin ^{2}\left(\frac{\theta}{2}\right) & \frac{\sin \theta}{\sqrt{2}} \\
\frac{\sin \theta}{\sqrt{2}} & \frac{\sin \theta}{\sqrt{2}} & \cos \theta
\end{array}\right)
$$

where $\theta$ is a parameter that completely define the scattering matrix. The scattered $\mathrm{rf}$ signals $\underline{V}^{-}$is related to the incident $\mathrm{rf}$ signals $\underline{V}^{+}$by

$$
\underline{V}^{-}=\underline{S} \underline{V^{+}}
$$

where $V_{i}^{ \pm}$represents incident/reflected rf signal from the $i$.th port. We terminate the third port so that all the scattered power off that port is completely reflected; i.e.,

$$
V_{3}^{+}=V_{3}^{-} e^{i \psi}
$$

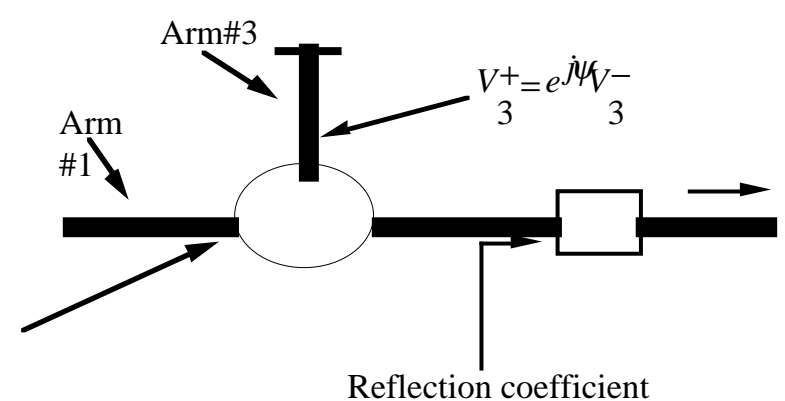

Three port device

Reflection coefficient represented by

Figure 1 Schematic diagram of the active switch.

By changing the angle $\psi$ of the third port terminator, the coupling between the first and the second ports can vary from 0 to 1 .

Let the reflection coefficient from the cascading two port junction equals to $x e^{j \phi}$. After some algebra one can show that the total reflection coefficient of the two cascaded junctions is

$$
\left|R_{\text {total }}(\psi)\right| \equiv\left|\frac{V_{1}^{-}}{V_{1}^{+}}\right|=\left(\frac{N_{1}}{D}\right)^{1 / 2}
$$


where

$$
D=2\left\{1+r_{3}^{2}-2 r_{3} \cos \psi+2\left(1-r_{3}\right) x \cos \left(\frac{\psi}{2}\right)\left[r_{3} \cos \left(\phi-\frac{\psi}{2}\right)-\cos \left(\phi+\frac{\psi}{2}\right)\right]+\left(1-r_{3}\right)^{2} x^{2} \cos ^{2}\left(\frac{\psi}{2}\right)\right\},
$$

and

$$
N_{1}=2\left\{\left(1-r_{3}\right)^{2} \cos ^{2}\left(\frac{\psi}{2}\right)+2\left(1-r_{3}\right) x \cos \left(\frac{\psi}{2}\right)\left[r_{3} \cos \left(\phi-\frac{\psi}{2}\right)-\cos \left(\phi+\frac{\psi}{2}\right)\right]+x^{2}\left[1+r_{3}^{2}-2 r_{3} \cos \psi\right]\right\} \text {. }
$$

and $r_{3} \equiv \cos \theta$ is the reflection coefficient from the third port when the other two ports are matched.

The outgoing wave in the third arm has an amplitude

$$
\left|V_{3}^{-}\right|=\left(\frac{N_{3}}{D}\right)^{1 / 2}\left|V_{1}^{+}\right|
$$

where

$$
N_{3}=\left(1-r_{3}\right)^{2}\left[2 x \cos \phi-x^{2}-1\right]
$$

\section{THE SILICON SWITCH}

To actively change the angle of the reflection coefficient at the third port we place a piece of semiconductor material in the third arm. An external stimulus such as an electron beam or a laser light can induce an electron-hole plasma layer at the surface of the semiconductor, thus changing its dielectric constant. Therefore, the propagation constant of rf signals through the active arm changes; and consequently the coupling between the other two ports also changes.

For the pulse compression system application associated with the NLC, it is required to change the reflection coefficient at the first arm between two fixed values[4]. The device should remain in one state for approximately $2 \mu \mathrm{sec}$, and in the other state for $250 \mathrm{nsec}$. Since silicon has a carrier life time that can extend from $1 \mu \mathrm{sec}$ to $1 \mathrm{msec}$ it seems like a natural choice for this application. One can excite the plasma layer with a very short pulse from the external stimulus $(\sim 5 \mathrm{nsec})$ and the device will stay in its new status long enough till all the rf signal is terminated. The repetition rate for this pulse compression system is $180 \mathrm{pulse} / \mathrm{sec}$. This will give enough time between pulses for the switch to completely recover.

Indeed, this switch need to have a very small amount of losses. Following classical arguments[5], one can show that the dielectric of constant of a semiconductor material is

$$
\varepsilon=\varepsilon_{0} \varepsilon_{r}\left(1-\sum_{i} \frac{X_{i}}{1-j Z_{i}}\right)
$$

where

$$
\begin{gathered}
X_{i}=\frac{N_{i} e^{2}}{\varepsilon_{0} \varepsilon_{r} m_{i}^{*} \omega^{2}}, \\
Z_{i}=\frac{v_{i}}{\omega},
\end{gathered}
$$

$\omega$ is the radial frequency of the rf signal, $m_{i}^{*}$ is the effective mass of carrier $i$ (electron, light hole and heavy hole), $N_{i}$ is carrier density, $\mathrm{e}$ is the electron charge, and $v_{i}$ is the collision frequency. This later quantity is related to the measured values of the dc. mobility $\mu_{i}[6]$ as follows:

$$
\frac{1}{v_{i}}=\frac{\mu_{i} m_{i}^{*}}{e}
$$

Comparison between estimates of $v_{i}$ for silicon to 11.424 $\mathrm{GHz}$, the operating frequency of the NLC, shows that $Z_{i} \gg>1$. Hence, one can show that the dielectric constant is given by the classical relation

$$
\varepsilon=\varepsilon_{0} \varepsilon_{r}\left(1-j \frac{\sigma}{\omega \varepsilon_{0} \varepsilon_{r}}\right) ;
$$

where

$$
\sigma=e \sum_{i} \mu_{i} N_{i},
$$

which is the conductivity of the semiconductor

To minimize the losses in the off state, i.e., when there is no plasma excited, we need to have a very pure semiconductor material such that the intrinsic carrier density is very small. In the $O n$ state, i.e., when the plasma layer is excited, the carrier density should be large enough so that the semiconductor acts like a good conductor and thus minimizing the losses.

At a carrier density $10^{19} / \mathrm{cm}^{3}$ silicon would have a conductivity of $\sim 3.3 \times 10^{3} \mathrm{mho} / \mathrm{cm}$. This is two orders of magnitude smaller than that of copper. However, it is high enough to make an effective reflector. The skin depth of $\mathrm{rf}$ signal at the NLC frequency at this conductivity level is $\sim 8 \mu \mathrm{m}$. In choosing the laser wavelength to produce the photoinduced carriers, light penetration depth should be comparable to this skin depth.

\section{HIGH POWER SWITCH FOR NLC}

To compress the rf signal efficiently by a factor of 8 the magnitude of the reflection coefficient of an iris need to change between 0.84 and 0.39 [4]. This change should take place in less than $5 \mathrm{nsec}$. This iris should be able to handle $300 \mathrm{MW}$ when it has a reflection coefficient of 0.84 for a period of 1.75 $\mu \mathrm{sec}$, and $1.9 \mathrm{GW}$ for a period of $250 \mathrm{nsec}$ when it has a reflection coefficient of 0.39 . The repetition rate for the whole process is $180 \mathrm{~Hz}$.

Consider making the active arm, in the device discussed in section III, from a circular waveguide operating at the fundamental mode $\left(T E_{11}\right)$. By placing a silicon wafer that has the same cross sectional area as the waveguide we can change the reflection coefficient angle $\psi$ between two values. At the off state the rf signal will be reflected from a short circuit that terminate the active arm. At the on state the signal will be reflected from the silicon wafer that acts like a good conductor because of the induced plasma layer.

The high power constrains on the switch discussed above now translates to the following two conditions: 
1. in the off state the silicon wafer should not be subjected to an electric field that exceed $150 \mathrm{kV} / \mathrm{cm}$ which is the estimated limit for the avalanche break down of a piece of silicon that has dimensions of few $\mathrm{cm}$.

2. At the on state, the amount of power dissipated in the silicon wafer should not raise its temperature so that it exceeds 70C when switch is to be operated at the off state once more.

Making the switch operate at the off state during $1.75 \mu \mathrm{sec}$ when the power levels are relatively low seems a natural choice. One can show that peak electric field in this arm in terms of the incident power, $P_{i n c}$, to the first port is

$$
E_{\max }=2.31\left[\varepsilon_{r}-0.0859\left(\frac{\lambda}{a}\right)^{2}\right]^{-1 / 4}\left(\frac{N_{3}}{D}\right)^{1 / 2} \frac{\left(Z_{0} P_{i n c}\right)^{1 / 2}}{a} \text {; }
$$

where $\lambda$ is the free space wavelength, $Z_{0}$ is the free space wave impedance, $a$ is the circular waveguide radius, and $\varepsilon_{r}$ is the dielectric constant of the material filling it.

By making the coupling to the third port as small as necessary one can reduce the peak field for any given amount of incident power. Also, increasing the diameter of the waveguide will reduce the field.

On the other hand, the power dissipated at the silicon wafer during the on state is

$$
P_{\text {losses }} / P_{\text {inc }}=4 \pi \frac{\delta}{\lambda}\left[\varepsilon_{r}-0.0859\left(\frac{\lambda}{a}\right)^{2}\right]^{1 / 2}\left(\frac{N_{3}}{D}\right) \text {; }
$$

where

$$
\delta=\left(\omega \mu_{0} \sigma / 2\right)^{-1 / 2},
$$

which is the skin depth of the rf signal inside the silicon wafer. Reducing the coupling to the third port increases the losses. Further, increasing the diameter also increase the losses.

Hence, the conditions for minimum peak field and small losses contradict each other. However, a reasonable compromise can be reached. For example, we choose the value of $r_{3}=0.866$, the value of $x=0.84$, the angle $\phi=23^{\circ}$, and the waveguide radius $\mathrm{a}=0.35^{\prime}$, we would have an active iris that have a peak electric field of less than $150 \mathrm{kV} / \mathrm{cm}$ in the active arm for an incident power of $500 \mathrm{MW}$, while maintaining a reflection coefficient of 0.84 . This is illustrated in Fig 2. At the on state, the losses in the third arm is less than $2 \%$, while maintaining a reflection coefficient of 0.39 . This is illustrated in Fig. 3.

To cool the silicon during the on state, we are currently investigating the possibility of using a diamond substrate.

\section{CONCLUSION}

We reported a three port device that can serve as an active iris for the NLC pulse compression system. We presented the design equations and design methodology for such an iris. We finally gave a design example for such an iris. We demonstrated the possibility of such a device to work at the high power levels required by the NLC rf pulse compression system.

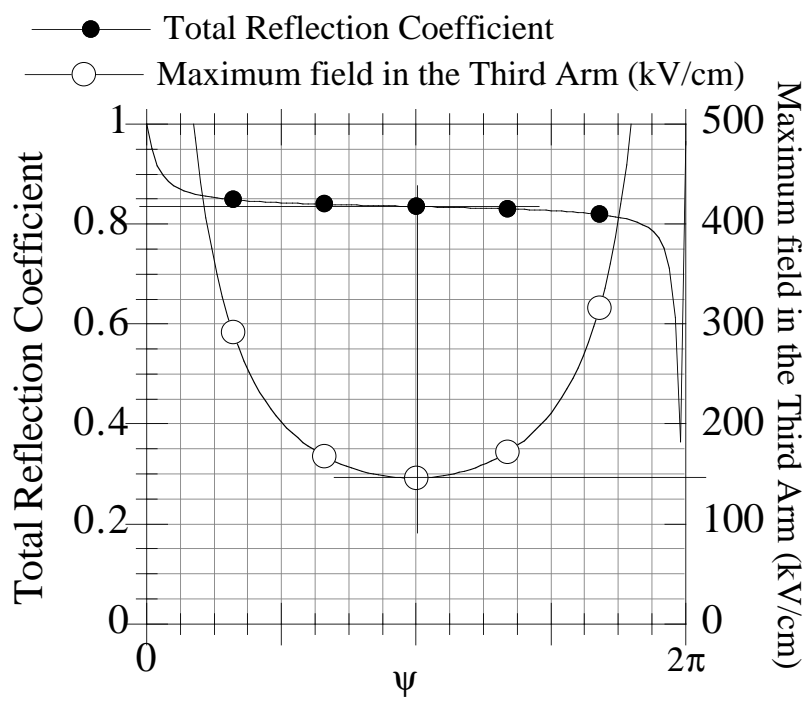

$\mathrm{F}$

Figure 2. Reflection coefficient and peak electric field versus the phase angle of the active port

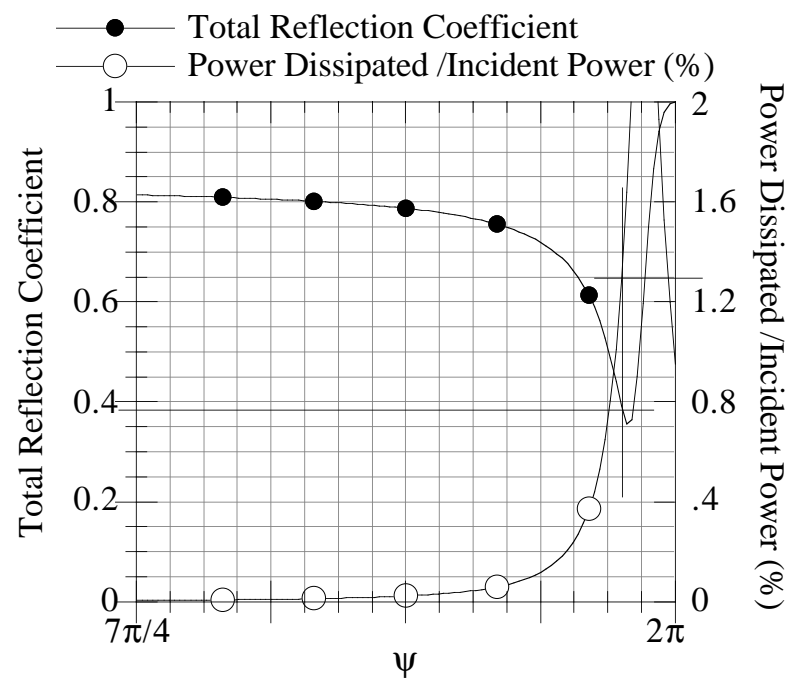

Figure 3. Reflection coefficient and power dissipated in the silicon wafer during the on state versus the phase of the active port.

\section{REFERENCES}

[1] R. D. Ruth et. al., "The Next Linear Collider Test Accelerator," Proc. of the IEEE Particle Accelerator Conference, Washington DC, May 1993, p. 543.

[2] Lee, H. Chi, Editor, "Picosecond optoelectronic devices, "Academic Press Inc., Orlando, 1984.

[3] Nunnally, W. C., and Hammond R. B., "Optoelectronic switch for pulsed power," Ch. 12 in Ref[2]

[4] Tantawi, S. G. , Ruth, R. D., Vlieks A. E., "Active pulse compression using resonant delay lines," This proceedings, also in SLAC-PUB 6748

[5] Wait, J. R. "Electromagnetics and plasmas," Holt, Rinehart and Winston, Inc. New York, 1968

[6] SZE S. M. "Physics of semiconductor devices, "John Wiley \& Sons, Inc., New York, 1969. 